\section{Maximizing quality in the manufacture of biologicals}

\author{
Gustavo Grampp, PhD; Sundar Ramanan, PhD
}

Biological product quality is susceptible to unexpected manufacturing issues, and the resulting variation may impact the safety or efficacy of these medicines and increase risks to patients. These risks can be managed more effectively by manufacturers' adoption of the culture and practices of high reliability organizations and by their sharing of quality risk management information.

Keywords: Biologicals, manufacturing, quality, safety

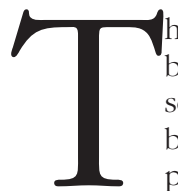
he manufacture of therapeutic biologicals is constantly evolving science. Early naturally sourced biologicals were extracted and purified from animal tissues, cadavers, or donated blood with attendant risks of unwanted immune responses or infection with source-derived viruses. Early recombinant biologicals contained impurities from the manufacturing process that were sometimes associated with immunogenicity [1].

Many of these risks have been mitigated through modern bioprocessing technology but the manufacture of biologicals today still faces significant challenges: how can we ensure that biological medicines maintain their high quality, from batch to batch, and are supplied without interruptions to patients who may require long-term therapy to manage their medical conditions?

Biologicals are sensitive to manufacturing process conditions, handling and changes Most biological medicines are manufactured using living cells that have been engineered to produce therapeutic proteins in large quantities. As these proteins are very sensitive to their conditions of synthesis and handling, a series of critical culturing and purification steps is required to produce a consistent, high quality active ingredient. The complexity of this process and the precision of required control require careful design and strict adherence to procedures as any changes introduced in the process can potentially influence the quality of the final product, including the structure, function and purity of the active ingredient [2].

The complexities of manufacturing biologicals also apply to biosimilars, which are approved on the basis of demonstrating highly similar quality, safety and efficacy to originator biologicals. In attempting to copy the originator product biosimilar manufacturers must independently design their own cell cultures and production steps. This is because the source materials, including the cell lines and the processes by which the original biologicals are made are confidential and may be protected by intellectual property laws, making exact replication of the steps extremely difficult, and typically resulting in structural differences between the products [3].

While many structural differences have no clinical relevance, it is possible that differences in protein folding, structural modifications (such as glycosylation), batch composition, and even the product container may have unexpected impact to the safety or efficacy of the product. For example, a product container contributed to a trend in immunogenicity for EPREX/ ERYPO (sold in the EU) starting in 1998. The immunogenicity trend manifested as a sharp increase in the incidence of pure red cell aplasia (PRCA) in patients with chronic

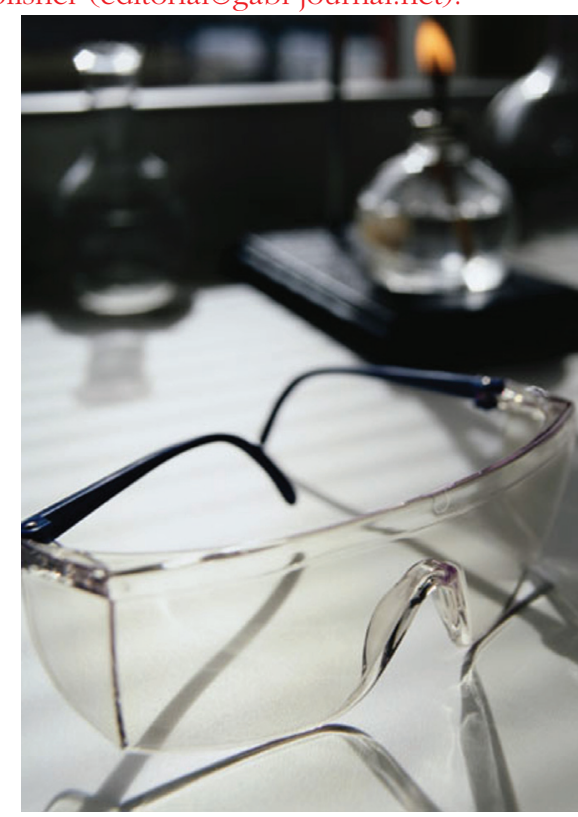

kidney disease and was ultimately traced to the effects of organic compounds leaching into the pre-filled syringes from uncoated rubber stoppers used by the manufacturer of EPREX/ERYPO. Due to the subtlety of the effect and the latency of the onset of PRCA (in some cases up to nine months after initiation of treatment) it took several years to identify and mitigate the effect of the organic leachate by changing to coated rubber stoppers [4]. Despite this discovery, new cases continue to emerge, generally in clusters. It is believed that the clusters were associated with protein aggregates potentially caused by a variety of factors including drug substance stability, improper handling, or interactions with container leachates [5].

The PRCA incident illustrates the importance of managing unexpected manufacturing events because the issue was not detected before patients were exposed to the impacted product. In many cases, manufacturing issues have been detected and investigated before the product was distributed to patients. These successful examples of managing unexpected events are known to individual manufacturers and to regulatory agencies, but are rarely publicized. Some examples that have been published include:

- Changes in glycosylation patterns due to altered cell culture conditions [6]

- Altered rates of biochemical modifications to the protein backbone after switching raw material supplier [6] 
- Turbidity in pre-filled syringes due to interaction of metal leachate with the protein product [7]

- A hard-to-detect microbial contaminant that managed to evade cell culture medium filtration and testing programmes [8]

- Presence of an impurity in the raw material used for a pegylated protein product [9]

\section{Lack of compliance with cGMP (current good manufacturing practices) affects} quality and reliable supply

Manufacturing and quality control issues like those just described can potentially impact patient safety and result in a loss of confidence in the quality of biologicals, but they can also impact to other products manufactured in the facility and cause product recalls and drug shortages, any of which can have profound effects on the company, customers, the biotech industry, providers and patients.

Regulatory agencies in the EU and US have recently emphasized the implications of this dynamic [10, 11]. Officials from the US Food and Drug Administration recently published an article describing quality management failures as a factor in sterile injectable drug shortages:

'... drug shortages are first and foremost driven by the inability of various firms to maintain production because of the failure of quality management in facilities that produce the finished dosage form of the drug ...',

\section{- J Woodcock and $M$ Wosinska, Center for Drug Evaluation and Research for the US Food and Drug Administration [11].}

Although FDA authors refer to the relationship of quality management to shortages of sterile injectable drugs, the issues cited are also relevant to the manufacture of biologicals. Biologicals share some of the same quality control issues with sterile injectables in that the final products are filled in facilities subject to similar stringency of control; but biologicals are, if anything, more susceptible to quality issues due to their relatively high sensitivity to multiple manufacturing steps. The rapidly increasing array of innovative biologicals and biosimilars could increase the chances of unexpected quality issues. These risks can be mitigated with investments in process and facility design, quality control systems, and management oversight, all of which can increase manufacturing reliability. While current market forces may not reward investments in quality and reliability, FDA may explore mechanisms to change this dynamic:

'... FDA could support the buyers and payers in their purchase and reimbursement decisions by providing them with meaningful manufacturing quality metrics. This general approach has been successfully used in many other settings where quality is difficult to observe or quality signals are difficult to interpret. Restaurant grades, HMO scorecards or even a US Pharmacopeia stamp on vitamins are just a few among many tools that utilize this concept ...' [11].

Recognition of high quality operations could also apply to the biologicals industry, and industry can anticipate this with increased investment in quality systems and sharing of best practices among peer manufacturers.

\section{Sharing best practices in manufacturing and quality}

The bioprocessing industry currently shares information about manufacturing challenges and product quality risks via congresses and publications. Regulators often contribute to this by sharing anonymous case studies and by encouraging companies to publish novel findings. For example, after learning of a number of examples of unexpected product quality impact from chemicals leaching from primary containers, FDA published a compilation of these case studies along with recommendations for best practices to evaluate such risks [12]. In furtherance of this objective, Amgen published its experience with a tungsten residual during final production that led to rejection of product during visual inspection [13].

To a large extent this type of sharing focuses on phenomenon with an element of scientific novelty, and it is less common for companies to publish manufacturing challenges and investigations that lack such novelty or that could raise legal issues. This void can be partially filled through participation in technical consortia where case studies and best practices can be shared less formally. Such consortia exist. For example, Rx-360 was established in 2009 to share best practices with raw material and component sourcing in an increasingly global supply chain [14] With regard to competitive concerns, we would draw a clear distinction between best practices that ensure patient safety and other proprietary information that might, for example, speed production, reduce costs and/or improve output.

\section{High reliability organizations offer a model for the industry}

Risks to product quality can be managed with focus on traditional GMP compliance and quality management, but manufacturers can also benefit from the practices of high reliability organizations (HROs). HROs invest in and engage well-trained and experienced support staff and advocate for management cultures that reward, '... in-depth analysis of unexpected results, robust risk assessments, and timely and effective implementation of mitigation measures' [6]. HROs exist where high performance is needed despite overwhelming potential for error and disaster. Examples of HROs in other fields include: nuclear power plants, aircraft carriers, emergency rooms, air traffic control stations, first responder protocols, and wilderness firefighters [15]. While regulators increasingly expect biologicals manufacturers to adapt risk management programmes including some of these behaviors, HROs set a standard for integration and prioritization of these practices that, if more widely adapted, could improve the industry's reliability and reputation - and further protect patients.

In addition to building a high reliability manufacturing culture, organizations can invest in a comprehensive strategy to reduce supply risks. Such a strategy could include multiple components [16, 17]:

- Prevention, e.g. compliance with or exceeding cGMP standards

- Technology, e.g. ensuring quality of raw materials using latest detection methods

- Inventory, e.g. ensuring adequate stock of drug in the event of natural disaster

- Diversification, e.g. multiple plants qualified for drug manufacture

In addition, a successful HRO will educate technical support staff to help orient and inform them so they can respond most effectively to unexpected manufacturing issues and incorporate findings into ongoing risk management. Critical background information includes the product's history, the current manufacturing environment and any analogous situations that may have been encountered within the company or by other manufacturers. The response team should have senior-level support and have access to the external resources they need, e.g. consultants and analyses; and be 
Biosimilars for Healthcare Professionals

authorized to examine every component of the manufacturing process, including in-house laboratories and manufacturing facilities and external vendors and suppliers, with the goal of conducting a focused and thorough investigation on the root causes of the unexpected event and identifying corrective actions [6].

Manufacturers that consistently strive to exceed minimum standards, that invest in supply risk mitigation, and that willingly adapt best practices shared by industry peers could benefit from an enhanced reputation as reliable suppliers.

\section{Conclusion}

Biosimilars, like all biologicals, are complex medicines produced in living cells and are highly sensitive to their manufacturing and processing conditions. Because of their complexity and sensitivity biologicals are at increased risk of quality issues compared with traditional drugs and generics, and this can affect the efficacy and safety of these treatments. Active participation in industry consortia where best practices can be shared among peers and a corporate focus on reliability of manufacturing can help minimize the risk and impact of unexpected quality issues, to the benefit of patients and companies.

\section{Acknowledgement}

Editorial assistance was provided by $\mathrm{Mr}$ Alex Brownstein of BioScience Communications, New York, NY, USA, whose work was funded by Amgen Inc.

Competing interests: Dr Gustavo Grampp and Dr Sundar Ramanan are employees of Amgen Inc and own stock in Amgen Inc.
Provenance and peer review: Not commissioned; externally peer reviewed.

\section{Co-author}

Sundar Ramanan, PhD, R \& D Policy, Amgen Inc, One Amgen Center Drive, Thousand Oaks, CA, USA.

\section{References}

1. Ryff JC. Clinical investigation of the immunogenicity of interferon-alpha 2a. J Interferon Cytokine Res. 1997;17 Suppl 1:S29-33.

2. Shellekens H. Biosimilar therapeutics-what do we need to consider? NDT Plus. 2009;2(Suppl 1): i27-36.

3. Hincal F. An introduction to safety issues in biosimilars/follow-on biopharmaceuticals. JMed CBR Def. 2009;7:1-18.

4. Boven K, Knight J, Bader F, Rossert J, Eckardt KU, Casadevall N. Epoetin-associated pure red cell aplasia in patients with chronic kidney disease: solving the mystery. Nephrol Dial Transplant. 2005;20 Suppl 3:iii33-40.

5. Macdougall IC, Roger SD, de Francisco A, Goldsmith DJ, Schellekens H, Ebbers H, et al. Antibody-mediated pure red cell aplasia in chronic kidney disease patients receiving erythropoiesis-stimulating agents: new insights. Kidney International. 2012;81:727-32.

6. Grampp G, Ramanan S. Managing unexpected events in the manufacturing of biologic medicines. BioDrugs. 2013 Aug;27(4):305-16.

7. Seidl A, Hainzl O, Richter M, Fischer R, Bohm S, Deutel B, et al. Tungsten-induced denaturation and aggregation of epoetin alfa during primary packaging as a cause of immunogenicity. Pharm Res. 2012 Jun;29(6):1454-67.

8. Chen J, Bergevin J, Kiss R, Walker G, Battistoni T, Lufburrow P, et al. Case study: a novel bacterial contamination in cell culture production Leptospira licerasiae. PDA J Pharm Sci Technol. 2012 Nov-Dec;66(6):580-91.
9. Zhang B, Towers EW, Poppe L, Cockrill SL. Analytical characterization of a novel degradation product in a PEGylated recombinant protein. J Pharm Sci. 2011;100(11):4607-16.

10. European Medicines Agency. Reflection paper on medicinal product supply shortages caused by manufacturing/good manufacturing practice compliance problems [homepage on the Internet]. 2012 [cited Sep 15]. Available from: http://www. ema.europa.eu/ema/index.jsp?curl=pages/includes/ document/document_detail.jsp?webContentId= WC500135113\&mid=WC0b01ac058009a3dc

11. Woodcock J, Wosinska M. Economic and technological drivers of generic sterile injectable drug shortages. Clin Pharmacol Ther. 2013;93(2): 170-6.

12. Markovic I. Evaluation of safety and quality impact of extractable and leachable substances in therapeutic biologic protein products: a risk-based perspective. Expert Opin Drug Saf. 2007;6(5): 487-91.

13. Jiang Y, Nashed-Samuel Y, Li C, Liu W, Pollastrini J, Mallard D, et al. Tungsten-induced protein aggregation: solution behavior. J Pharm Sci. 2009;98(12): 4695-720.

14. Rx-360: an International Pharmaceutical Supply Chain Consortium [homepage on the Internet]. 2013 [cited 2013 Sep 18]. Available from: http:// www.rx-360.org/Home/tabid/38/Default.aspx

15. Weick KE, Sutcliffe KM. Managing the unexpected: resilient performance in an age of uncertainty. 2nd ed. San Francisco: Wiley; 2007.

16. Mica A, Green L. Drug availability: considerations for the hospital pharmacist. Eur J Hosp Pharm. 2012;19:160-1.

17. Mica A, et al. Steps to ensure adequate supply of biological medicines: considerations for the healthcare provider. Generics and Biosimilars Initiative Journal (GaBI Journal) 2013;2(3):136-43. doi:10.5639/gabij.2013.0203.038

DOI: 10.5639/gabij.2013.0204.049

Copyright @ 2013 Pro Pharma Communications International 